\title{
SOME CLOSED FORMULAS AND CONJECTURES FOR HODGE INTEGRALS
}

\author{
JIAN ZHOU
}

\begin{abstract}
We announce the proof of some closed formulas for Hodge integrals, including some low degree cases of a remarkable formula conjectured by Marino and Vafa based on string duality, and a conjecture by Monni, Song, and Song. We also make some conjectures based on the proved formulas.
\end{abstract}

Recently there has been much work on Hodge integrals $[7,3,1,8,9,18]$. Recall on the compactified moduli space $\overline{\mathcal{M}}_{g, n}$, besides the line bundles $L_{i}$ whose fibers at a stable curve with marked points $\left(C, x_{1}, \ldots, x_{n}\right)$ are $T_{x_{i}}^{*} C$, there is the Hodge bundle $\mathbb{E}$ whose fiber is $H^{0}\left(C, \omega_{C}\right)$. Their Chern classes provide some naturally defined classes on $\overline{\mathcal{M}}_{g, n}$ :

$$
\begin{array}{rlrl}
\psi_{i} & =c_{1}\left(L_{i}\right), & i=1, \ldots, n, \\
\lambda_{j}=c_{j}(\mathbb{E}), & j=1, \ldots, g .
\end{array}
$$

An integral of the form

$$
\int_{\overline{\mathcal{M}}_{g, n}} \psi_{1}^{j_{1}} \cdots \psi_{n}^{j_{n}} \lambda_{1}^{k_{1}} \cdots \lambda_{g}^{k_{g}}
$$

is called a Hodge integral. They can be reduced to integrals of the form

$$
\int_{\overline{\mathcal{M}}_{g, n}} \psi_{1}^{j_{1}} \cdots \psi_{n}^{j_{n}}
$$

whose evaluations are given by the Witten conjecture/Kontsevich's theorem $[19$, 10, 17], and a Maple program is available to carry out the calculations [2].

It is very interesting to have closed formulas for Hodge integrals. Such integrals naturally arise when one computes Gromov-Witten invariants by localization techniques $[11,7]$ in the higher genus case. Closed formulas for all genera are then a very important ingredient in such calculations. We have found the following generating series for Hodge integrals particularly interesting:

$$
C_{n_{1}, \ldots, n_{h}}(\lambda ; \alpha, \beta)=\sum_{g \geq 0} \lambda^{2 g} \int_{\overline{\mathcal{M}}_{g, h}} \frac{\Lambda_{g}^{\vee}(1) \Lambda_{g}^{\vee}(\alpha) \Lambda_{g}^{\vee}(\beta)}{\prod_{i=1}^{h} \frac{1}{n_{i}}\left(\frac{1}{n_{i}}-\psi_{i}\right)},
$$

Received January 3, 2003.

This research is partially supported by research grants from NSFC and Tsinghua University. 
where $\Lambda_{g}^{\vee}(\alpha)=\sum_{i=0}^{g}(-1)^{i} \alpha^{g-i} \lambda_{i}$. Here the leading term is

$$
\left(\sum_{i=1}^{h} n_{i}\right)^{h-3} \cdot \prod_{i=1}^{h} n_{i}^{2}
$$

in all cases. (We will often write $C_{n_{1}, \ldots, n_{h}}(\lambda ; \alpha, \beta)$ as $C_{n_{1}, \ldots, n_{h}}(\alpha, \beta)$ for simplicity of notations.) For example, by Mumford's relations [16]:

$$
\Lambda_{g}^{\vee}(\alpha) \Lambda_{g}^{\vee}(-\alpha)=(-1)^{g} \alpha^{2 g},
$$

one gets:

$$
C_{n_{1}, \ldots, n_{h}}(\lambda ; p,-p)=\prod_{i=1}^{h} n_{i}^{2} \cdot \sum_{g \geq 0}(-1)^{g}(p \lambda)^{2 g} \int_{\overline{\mathcal{M}}_{g, h}} \frac{\Lambda_{g}^{\vee}(1)}{\prod_{i=1}^{h}\left(1-n_{i} \psi_{i}\right)} ;
$$

on the other hand one has the ELSV formula $[1,8]$ :

$$
\mu^{g}\left(n_{1}, \ldots, n_{h}\right)=\frac{\left(2 g-2+\sum_{i=1}^{h} n_{i}+h\right) !}{\left|\operatorname{Aut}\left(n_{1}, \ldots, n_{h}\right)\right|} \prod_{i=1}^{h} \frac{n_{i}^{n_{i}}}{n_{i} !} \int_{\overline{\mathcal{M}}_{g, h}} \frac{\Lambda_{g}^{\vee}(1)}{\prod_{i=1}^{h}\left(1-n_{i} \psi_{i}\right)}
$$

Hence $C_{n_{1}, \ldots, n_{h}}(\lambda ; p,-p)$ is essentially the generating series of the Hurwitz numbers $\mu^{g}\left(n_{1}, \ldots, n_{h}\right)$.

In a series of papers $[3,4,5]$, many closed formulas for Hodge integrals have been found. We mention here the following one which is most relevant to this work:

$$
1+\sum_{g>0} \lambda^{2 g} \sum_{i=0}^{g} k^{i} \int_{\overline{\mathcal{M}}_{g, 1}} \psi_{1}^{2 g-2+i} \lambda_{g-i}=\left(\frac{\lambda / 2}{\sin (\lambda / 2)}\right)^{k+1} .
$$

In [18], the following generalization was made:

$$
C_{1}(\lambda ; \alpha, \beta)=1+\sum_{g>0} \lambda^{2 g} \int_{\overline{\mathcal{M}}_{g, 1}} \frac{\Lambda_{g}^{\vee}(1) \Lambda_{g}^{\vee}(\alpha) \Lambda_{g}^{\vee}(\beta)}{1-\psi_{1}}=\left(\frac{\sin (\lambda / 2)}{\lambda / 2}\right)^{\alpha+\beta} .
$$

Using Mumford's relations, one recovers (3) by taking $\alpha=-1, \beta=-k$. By taking $\alpha=p$ and $\beta=-p-1$, one obtains by (4):

$$
C_{1}(\lambda ; p,-p-1)=\frac{\lambda / 2}{\sin (\lambda / 2)} .
$$

As shown in [20], this is a special case of a remarkable formula for $C_{n_{1}, \ldots, n_{h}}(\lambda$; $p,-p-1)$ conjectured by Marino and Vafa, which we now turn to.

Based on conjectural duality with Chern-Simons theory with Wilson loop observables, Marino and Vafa [14] obtained the following formula for the free 
energy of the open string theory for $\mathcal{O}(-1) \oplus \mathcal{O}(-1) \rightarrow \mathbb{P}^{1}$ :

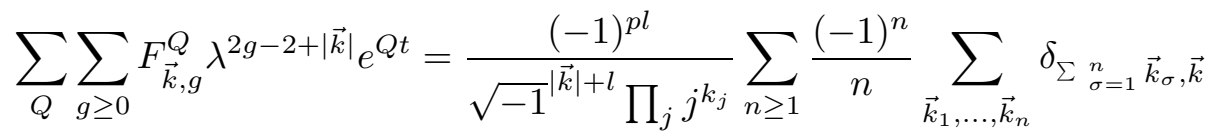

$$
\begin{aligned}
& \cdot \sum_{R_{\sigma}} \prod_{\sigma=1}^{n} \frac{\chi_{R_{\sigma}}\left(C\left(\vec{k}_{\sigma}\right)\right)}{z_{\vec{k}_{\sigma}}} \cdot e^{\sqrt{-1} p \kappa_{R_{\sigma}} \lambda / 2} \\
& \cdot \prod_{1 \leq i<j \leq c_{R_{\sigma}}} \frac{\sin \left[\left(l_{i}^{\sigma}-l_{j}^{\sigma}+j-i\right) \lambda / 2\right]}{\sin [(j-i) \lambda / 2]} \\
& \cdot \prod_{i=1}^{c_{R_{\sigma}}} \frac{\prod_{v=-i+1}^{l_{i}^{\sigma}-i}\left(e^{\frac{t}{2}+\frac{\sqrt{-1} v \lambda}{2}}-e^{-\frac{t}{2}-\frac{\sqrt{-1} v \lambda}{2}}\right)}{\prod_{v=1}^{l_{i}^{\sigma}} 2 \sin \left[\left(v-i+c_{R_{\sigma}}\right) \lambda / 2\right]} .
\end{aligned}
$$

Some explanation of the notations are needed here. For a partition $R$ given by

$$
l_{1} \geq l_{2} \geq \cdots \geq l_{c_{R}}>0
$$

$\chi_{R}$ denotes the character of the irreducible representation of $S_{l}$, indexed by $R$, where

$$
l=\sum_{i=1}^{c_{R}} l_{i}
$$

The number $\kappa_{R}$ is defined by:

$$
\kappa_{R}=l+\sum_{i}\left(l_{i}^{2}-2 i l_{i}\right)
$$

For each positive integer $i$,

$$
k_{i}=\left|\left\{l_{j}: l_{j}=i\right\}\right| \text {. }
$$

The vector $\vec{k}$ is just $\left(k_{1}, k_{2}, \ldots\right)$, and $C(\vec{k})$ denotes the conjagacy class of $S_{l}$ corresponding to the partition

$$
1^{k_{1}} 2^{k_{2}} \cdots
$$

and $\chi_{R}(C(\vec{k}))$ is the value of the character on the conjugacy class $C(\vec{k})$, i.e., the value of the character on any element in that conjugacy class. Furthermore,

$$
z_{\vec{k}}=\prod_{j} k_{j} ! j^{k_{j}}
$$

According to [9] the leading term of (5) is

$$
\sum_{g \geq 0} \lambda^{2 g}(p(p+1))^{h-1} \prod_{i=1}^{h} \frac{\prod_{j=1}^{n_{i}-1}\left(j+n_{i} p\right)}{\left(n_{i}-1\right) !} \int_{\overline{\mathcal{M}}_{g, h}} \frac{\Lambda_{g}^{\vee}(t) \Lambda_{g}^{\vee}((-p-1) t) \Lambda_{g}^{\vee}(p t) t^{2 h-3}}{\prod_{i=1}^{h}\left(t-n_{i} \psi_{i}\right)}
$$


or by Lemma 2.1 in [18],

(6) $\sum_{g \geq 0} \lambda^{2 g}(p(p+1))^{h-1} \cdot \prod_{i=1}^{h} \frac{\prod_{j=1}^{n_{i}-1}\left(j+n_{i} p\right)}{\left(n_{i}-1\right) !} \cdot \int_{\overline{\mathcal{M}}_{g, h}} \frac{\Lambda_{g}^{\vee}(1) \Lambda_{g}^{\vee}(-p-1) \Lambda_{g}^{\vee}(p)}{\prod_{i=1}^{h}\left(1-n_{i} \psi_{i}\right)}$,

i.e., a multiple of $C_{n_{1}, \ldots, n_{h}}(\lambda ; p,-p-1)$. As pointed out in [14], by comparing (6) and (5), one obtains a conjectural formula for $C_{n_{1}, \ldots, n_{h}}(p,-p-1)$. In [20] this formula is explicitly written down as follows:

$$
\begin{aligned}
& C_{n_{1}, \ldots, n_{h}}(\lambda ; p,-p-1) \\
& =\lambda^{2-|\vec{k}|} \cdot \frac{1}{(p(p+1))^{h-1}} \cdot \prod_{i=1}^{h} \frac{n_{i} \cdot n_{i} !}{\prod_{j=1}^{n_{i}-1}\left(j+n_{i} p\right)} \cdot \frac{\prod_{j} k_{j} !}{\sqrt{-1}|\vec{k}|+l} \cdot \sum_{n \geq 1} \frac{(-1)^{n}}{n} \\
& \quad \sum_{\sum \sum_{\sigma=1}^{n} \vec{k}_{\sigma}=\vec{k}^{\sum} \sum_{\sigma=1}^{n}\left|R_{\sigma}\right|=l} \prod_{\sigma=1}^{n} \frac{\chi_{R_{\sigma}}\left(C\left(\vec{k}_{\sigma}\right)\right)}{z_{\vec{k}_{\sigma}}} \cdot e^{\sqrt{-1}\left(p+\frac{1}{2}\right) \kappa_{R_{\sigma}} \lambda / 2} \cdot V\left(R_{\sigma}\right),
\end{aligned}
$$

where

(8) $\quad V(R)=\prod_{1 \leq i<j \leq c_{R}} \frac{\sin \left[\left(l_{i}-l_{j}+j-i\right) \lambda / 2\right]}{\sin [(j-i) \lambda / 2]}$

$$
\cdot \frac{1}{\prod_{i=1}^{c_{R}} \prod_{v=1}^{l_{i}} 2 \sin \left[\left(v-i+c_{R}\right) \lambda / 2\right]} .
$$

The following result gives a much simpler expression for $V(R)$ :

Theorem 1. [20] Denote also by $R$ the Young diagram of the partition $R$ of $l$. For any square $x \in R$, denote by $h(x)$ the hook length of $x$. Then we have

$$
V(R)=\frac{1}{2^{l} \prod_{x \in R} \sin [h(x) \lambda / 2]} .
$$

Theorem 2. [21] One has

$$
\begin{aligned}
& C_{1}(\lambda ; p,-p-1)=\frac{\lambda / 2}{\sin (\lambda / 2)}, \\
& C_{2}(\lambda ; p,-p-1)=\frac{\lambda}{2 p+1} \frac{\sin [(2 p+1) \lambda / 2]}{\sin (\lambda / 2) \sin \lambda} \\
& C_{1,1}(\lambda ; p,-p-1)=\frac{1}{p(p+1)} \frac{\sin (p \lambda / 2) \sin [(p+1) \lambda / 2]}{\sin (\lambda / 2) \sin \lambda} \\
& C_{3}(\lambda ; p,-p-1)=\frac{3 \lambda}{(3 p+1)(3 p+2)} \cdot \frac{\sin [(3 p+1) \lambda / 2] \sin [(3 p+2) \lambda / 2]}{\sin (\lambda / 2) \sin \lambda \sin (3 \lambda / 2)} \\
& C_{2,1}(-p-1, p)=\frac{1}{p(p+1)\left(p+\frac{1}{2}\right)} \cdot \frac{\sin (p \lambda) \sin \left[\left(p+\frac{1}{2}\right) \lambda\right] \sin [(p+1) \lambda]}{\sin (\lambda / 2) \sin \lambda \sin (3 \lambda / 2)} \\
& C_{1,1,1}(-p-1, p)=\frac{4}{p^{2}(p+1)^{2} \lambda} \cdot \frac{\sin ^{2}(p \lambda / 2) \sin { }^{2}[(p+1) \lambda / 2]}{\sin (\lambda / 2) \sin \lambda \sin (3 \lambda / 2)} \\
& \cdot[\cos [(p+1 / 2) \lambda]+2 \cos (\lambda / 2)]
\end{aligned}
$$


They all match with the Marino-Vafa formula (7).

The formulas predicted by the Marino-Vafa formula are in general not of the above form. Some elementary algebraic manipulations are needed to transfer them to the present form which helps us to formulate some conjectures stated below. For example, Marino-Vafa formula yields:

$$
C_{1,1}(p,-p-1)=\frac{2}{p(p+1)}\left(-\frac{\cos \left[\left(p+\frac{1}{2}\right) \lambda\right]}{4 \sin (\lambda / 2) \sin \lambda}+\frac{1}{8 \sin ^{2}(\lambda / 2)}\right) .
$$

We convert it to the form in (11) as follows.

$$
\begin{aligned}
C_{1,1}(p,-p-1) & =\frac{2}{p(p+1)} \frac{-2 \sin (\lambda / 2) \cos \left(\left[p+\frac{1}{2}\right) \lambda\right]+\sin \lambda}{8 \sin ^{2}(\lambda / 2) \sin \lambda} \\
& =\frac{1}{2 p(p+1)} \frac{-\cos \left[\left(p+\frac{1}{2}\right) \lambda\right]+\cos (\lambda / 2)}{\sin (\lambda / 2) \sin \lambda} \\
& =\frac{1}{p(p+1)} \frac{\sin [(p+1) \lambda / 2] \sin (p \lambda / 2)}{\sin (\lambda / 2) \sin \lambda} .
\end{aligned}
$$

By inspecting (9), (10), and (12), we make the following:

Conjecture 1. For positive integer $n$ and any complex number $p$, one has

$$
C_{n}(\lambda ; p,-p-1)=n ! \frac{\lambda / 2}{\sin (\lambda / 2)} \prod_{i=1}^{n-1} \frac{\sin [(n p+i) \lambda / 2]}{(n p+i) \sin [(i+1) \lambda / 2]} .
$$

Here we give one more evidence for (15). Taking $p=0$, then (15) becomes:

$$
C_{n}(\lambda ; 0,-1)=\frac{n \lambda / 2}{\sin (n \lambda / 2)} .
$$

This matches with (3). Indeed, by Mumford's relations,

$$
\begin{aligned}
C_{n}(\lambda ; 0,-1) & =\sum_{g \geq 0} \lambda^{2 g} \int_{\overline{\mathcal{M}}_{g, 1}} \frac{\Lambda_{g}^{\vee}(1) \Lambda_{g}^{\vee}(-1) \Lambda_{g}^{\vee}(0)}{\frac{1}{n}\left(\frac{1}{n}-\psi_{1}\right)} \\
& =\sum_{g \geq 0} \lambda^{2 g} \int_{\overline{\mathcal{M}}_{g, 1}} \frac{\lambda_{g}}{\frac{1}{n}\left(\frac{1}{n}-\psi_{1}\right)}=\sum_{g \geq 0}(n \lambda)^{2 g} \int_{\overline{\mathcal{M}}_{g, 1}} \frac{\lambda_{g}}{1-\psi_{1}} \\
& =\frac{n \lambda / 2}{\sin (n \lambda / 2)} .
\end{aligned}
$$

Note (15) is not obvious from Marino-Vafa formula which predicts:

$$
\begin{aligned}
& C_{n}(\lambda ; p,-p-1) \\
& \quad=-\frac{n \cdot n !}{\prod_{j=1}^{n-1}(j+n p)} \cdot \frac{\lambda}{\sqrt{-1}^{n+1}} \sum_{|R|=n} \frac{\chi_{R}(n)}{n} \cdot e^{\sqrt{-1}\left(p+\frac{1}{2}\right) \kappa_{R} \lambda / 2} \cdot V(R) .
\end{aligned}
$$

Comparing (15) with (17), we get the following: 
Conjecture 2. For positive integer $n$ and any complex number $p$, one has

$$
\begin{array}{rl}
-\frac{\lambda}{\sqrt{-1}^{n+1}} \sum_{|R|=n} \chi_{R}(n) e^{\sqrt{-1}\left(p+\frac{1}{2}\right) \kappa_{R} \lambda / 2} & V(R) \\
& =\frac{\lambda / 2}{\sin (\lambda / 2)} \prod_{i=1}^{n-1} \frac{\sin [(n p+i) \lambda / 2]}{\sin [(i+1) \lambda / 2]} .
\end{array}
$$

In particular,

$$
-\frac{\lambda}{\sqrt{-1}^{n+1}} \sum_{|R|=n} \chi_{R}(n) e^{\sqrt{-1} \kappa_{R} \lambda / 4} V(R)=\frac{\lambda / 2}{\sin (n \lambda / 2)} .
$$

This conjecture has been verified up to $n=4$. It is interesting to find any relationship of (18) and (19) with constant term identities [13]. Formulas (11) and (13) provide evidence for the following

Conjecture 3. For positive integer $n$ and any complex number $p$, one has

$$
C_{n, 1}(\lambda ; p,-p-1)=n^{2} \cdot n ! \prod_{i=0}^{n} \frac{\sin [(n p+i) \lambda / 2]}{(n p+i) \sin [(i+1) \lambda / 2]}
$$

By comparing (20) with the Marino-Vafa formula, one gets for $n \geq 2$,

$$
\begin{aligned}
& \frac{1}{p(p+1)} \cdot \frac{n \cdot n !}{\prod_{j=1}^{n-1}(j+n p)} \cdot \frac{1}{\sqrt{-1}^{n+3}} \\
& \cdot\left(-\sum_{|R|=n+1} \frac{\chi_{R}(n, 1)}{n} \cdot e^{\sqrt{-1}\left(p+\frac{1}{2}\right) \kappa_{R} \lambda / 2} \cdot V(R)+\frac{1}{2} \cdot 2 \cdot \frac{1}{2 \sin (\lambda / 2)}\right. \\
&\left.\cdot \sum_{|R|=n} \frac{\chi_{R}(n)}{n} \cdot e^{\sqrt{-1}\left(p+\frac{1}{2}\right) \kappa_{R} \lambda / 2} \cdot V(R)\right) \\
&=n^{2} \cdot n ! \prod_{i=0}^{n} \frac{\sin [(n p+i) \lambda / 2]}{(n p+i) \sin [(i+1) \lambda / 2]}
\end{aligned}
$$

Combining with (18), one gets a conjecture similar to Conjecture 2.

Conjecture 4. For $n \geq 2$, one has

$$
\begin{aligned}
& \frac{-1}{\sqrt{-1}^{n+3}} \sum_{|R|=n+1} \chi_{R}(n, 1) \cdot e^{\sqrt{-1}\left(p+\frac{1}{2}\right) \kappa_{R} \lambda / 2} \cdot V(R) \\
& =\prod_{i=0}^{n} \frac{\sin [(n p+i) \lambda / 2]}{\sin [(i+1) \lambda / 2]}-\frac{1}{4 \sin ^{2}(\lambda / 2)} \prod_{i=1}^{n-1} \frac{\sin [(n p+i) \lambda / 2]}{\sin [(i+1) \lambda / 2]} .
\end{aligned}
$$


In particular,

$$
\frac{1}{\sqrt{-1}^{n+3}} \sum_{|R|=n+1} \chi_{R}(n, 1) \cdot e^{\sqrt{-1} \kappa_{R} \lambda / 4} \cdot V(R)=\frac{1}{4 \sin (\lambda / 2) \sin (n \lambda / 2)} .
$$

We have proved in [21] the following results which contains (19) and (22) as special cases:

Theorem 3. For any positive integer $n$ and any partition $\rho$ of $n$ we have

$$
\sum_{|\eta|=n} \frac{\chi_{\eta}(\rho) e^{\sqrt{-1} \kappa_{\eta} \lambda / 4}}{\prod_{x \in \eta} 2 \sin (h(x) \lambda / 2)}=\frac{\sqrt{-1}^{n-l(\rho)}}{2^{l(\rho)} \prod_{k} \sin ^{m_{k}(\rho)}(k \lambda / 2)} .
$$

We have some extra evidence for $(20)$ when $p=0$. Indeed, on the one hand, (20) predicts:

$$
C_{n, 1}(0,-1)=\frac{n^{2}}{n+1} \cdot \frac{(n+1) \lambda / 2}{\sin [(n+1) \lambda / 2]}
$$

on the other hand, one can calculate $C_{n, 1}(0,-1)$ directly using Mumford's relations, (3) and the following formula proved in [5]:

$$
\int_{\overline{\mathcal{M}}_{g, n}} \psi_{1}^{j_{1}} \cdots \psi_{n}^{j_{n}} \lambda_{g}=\left(\begin{array}{c}
2 g+n-3 \\
j_{1}, \ldots, j_{n}
\end{array}\right) \int_{\overline{\mathcal{M}}_{g, 1}} \frac{\lambda_{g}}{1-\psi_{1}} .
$$

Both (16) and (24) are special cases of the following:

Lemma 1. For any partition $\left(n_{1}, \ldots, n_{h}\right)$ of $d$, we have the following identity:

$$
C_{n_{1}, \ldots, n_{h}}(0,-1)=\prod_{i=1}^{h} n_{i}^{2} \cdot d^{h-3} \cdot \frac{d \lambda / 2}{\sin (d \lambda / 2)} .
$$

Proof. We have

$$
\begin{aligned}
& C_{n_{1}, \ldots, n_{h}}(0,-1) \\
= & \sum_{g \geq 0} \lambda^{2 g} \int_{\overline{\mathcal{M}}_{g, h}} \frac{\lambda_{g}}{\prod_{i=1}^{h} \frac{1}{n_{i}}\left(\frac{1}{n_{i}}-\psi_{i}\right)} \\
= & \prod_{i=1}^{h} n_{i}^{2} \sum_{g \geq 0} \lambda^{2 g} \sum_{j_{1}+\cdots+j_{h}=2 g+h-3} \int_{\overline{\mathcal{M}}_{g, h}} \lambda_{g} \cdot \prod_{i=1}^{h}\left(n_{i} \psi_{i}\right)^{j_{i}} \\
= & \prod_{i=1}^{h} n_{i}^{2} \sum_{g \geq 0} \lambda^{2 g} \sum_{j_{1}+\cdots+j_{h}=2 g+h-3}\left(\begin{array}{c}
2 g+h-3 \\
j_{1}, \ldots, j_{n}
\end{array}\right) \prod_{i=1}^{h} n_{i}^{j_{i}} \int_{\overline{\mathcal{M}}_{g, 1}} \frac{\lambda_{g}}{1-\psi_{1}} \\
= & \prod_{i=1}^{h} n_{i}^{2} \cdot \sum_{g \geq 0} \lambda^{2 g}\left(n_{1}+\cdots+n_{h}\right)^{2 g+h-3} \int_{\overline{\mathcal{M}}_{g, 1}} \frac{\lambda_{g}}{1-\psi_{1}} \\
= & \prod_{i=1}^{h} n_{i}^{2} \cdot d^{h-3} \cdot \frac{d \lambda / 2}{\sin (d \lambda / 2)} .
\end{aligned}
$$


By comparing (26) with (7), one is led to the following highly nontrivial combinatorial identity which we prove in [21]:

Theorem 4. One has

$$
\begin{array}{r}
\lim _{p \rightarrow 0} \lambda^{2-h} \cdot \frac{1}{(p(p+1))^{h-1}} \cdot \prod_{i=1}^{h} \frac{n_{i} \cdot n_{i} !}{\prod_{j=1}^{n_{i}-1}\left(j+n_{i} p\right)} \cdot \frac{\prod_{j} k_{j} !}{\sqrt{-1}|\vec{k}|+l} \cdot \sum_{n \geq 1} \frac{(-1)^{n}}{n} \\
\cdot \sum_{\substack{n \\
\sigma=1}} \sum_{\vec{k}_{\sigma}=\vec{k}_{\sum} \sum_{\sigma=1}^{n}\left|R_{\sigma}\right|=l} \prod_{\sigma=1}^{n} \frac{\chi_{R_{\sigma}}\left(C\left(\vec{k}_{\sigma}\right)\right)}{z_{\vec{k}_{\sigma}}} \cdot e^{\sqrt{-1}\left(p+\frac{1}{2}\right) \kappa_{R_{\sigma}} \lambda / 2} \cdot V\left(R_{\sigma}\right) \\
=\prod_{i=1}^{h} n_{i}^{2} \cdot d^{h-3} \cdot \frac{d \lambda / 2}{\sin (d \lambda / 2)}
\end{array}
$$

We now say a few words about the method used to prove Theorem 2. It is in a sense a generalization of the method in [3] and [18], but the details are much more complicated. In those references, localization on $\overline{\mathcal{M}}_{g, 0}\left(\mathbb{P}^{1}, 1\right)$ is used. We obtain our results by localization on $\overline{\mathcal{M}}_{g, 0}\left(\mathbb{P}^{1}, 2\right)$ and $\overline{\mathcal{M}}_{g, 0}\left(\mathbb{P}^{1}, 3\right)$. In degree 2 , we have the following technical result:

Theorem 5. [20] One has the following three identities:

$$
\begin{aligned}
& -C_{2}(\alpha, \beta) C_{2}(-\alpha,-\beta)+C_{1}(\alpha, \beta)^{2} C_{1,1}(-\alpha,-\beta) \\
& \quad+C_{1}(-\alpha,-\beta)^{2} C_{1,1}(\alpha, \beta)+\lambda^{2} \alpha \beta C_{1,1}(\alpha, \beta) C_{1,1}(-\alpha,-\beta)=0
\end{aligned}
$$

$$
\left(\beta+\frac{1}{2}\right) C_{2}(\alpha, \beta) C_{2}(-\alpha,-\beta-1)-(\beta+1) C_{1}(\alpha, \beta)^{2} C_{1,1}(-\alpha,-\beta-1)
$$

$-\beta C_{1}(-\alpha,-\beta-1)^{2} C_{1,1}(\alpha, \beta)-\beta \lambda^{2} \alpha(\beta+1) C_{1,1}(\alpha, \beta) C_{1,1}(-\alpha,-\beta-1)=0$.

$$
\begin{aligned}
& -\left(\alpha+\frac{1}{2}\right)\left(\beta+\frac{1}{2}\right) C_{2}(\alpha, \beta) C_{2}(-\alpha-1,-\beta-1) \\
& \quad+(\alpha+1)(\beta+1) C_{1}(\alpha, \beta)^{2} C_{1,1}(-\alpha-1,-\beta-1) \\
& \quad+\alpha \beta C_{1}(-\alpha-1,-\beta-1)^{2} C_{1,1}(\alpha, \beta) \\
& \quad+\lambda^{2} \alpha \beta(\alpha+1)(\beta+1) C_{1,1}(\alpha, \beta) C_{1,1}(-\alpha-1,-\beta-1) \\
& \quad=\frac{1}{4}\left(\frac{\lambda}{\sin \lambda}\right)^{2}
\end{aligned}
$$

The four terms on the left-hand side of each of the above formulas correspond to the four types of fixed point components parameterized by the following four types of decorated graphs:
(1) 2 (1)
(1) 1 (1) 1 (0)
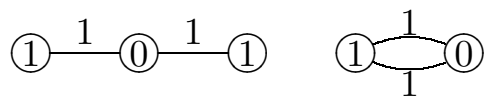
The applications of Theorem 5 requires closed formulas for $C_{n_{1}, \ldots, n_{h}}(\lambda ; p,-p)$. This can be achieved by exploiting the relationship (2) with Hurwitz numbers $[1,8]$. In [6] and [12], a partial differential equation called the cut-and-join equations have been proved for generating series of Hurwitz numbers. In [21] we observe that by considering the generating series of disconnected Hurwitz numbers, one can reformulate the cut-and-joint equation as a sequence of systems of linear ordinary differential equations, one for each degree. The initial values for these systems are very easy to obtain, hence one can explicitly solve the systems. By this method the author has obtained the following identities in [21]:

$$
\begin{aligned}
& \sum_{g \geq 0} \lambda^{2 g} \int_{\overline{\mathcal{M}}_{g, 1}} \frac{\Lambda_{g}^{\vee}(1)}{1-\psi_{1}}=1, \\
& \sum_{g \geq 0} \lambda^{2 g} \int_{\overline{\mathcal{M}}_{g, l}} \frac{\Lambda_{g}^{\vee}(1)}{\frac{1}{2}\left(\frac{1}{2}-\psi_{i}\right)}=\frac{\sinh \lambda}{\lambda} \\
& \sum_{g \geq 0} \lambda^{2 g} \int_{\overline{\mathcal{M}}_{g, 2}} \frac{\Lambda_{g}^{\vee}(1)}{\left(1-\psi_{1}\right)\left(1-\psi_{2}\right)}=\frac{1}{2}\left(\frac{\sinh (\lambda / 2)}{\lambda / 2}\right)^{2}, \\
& \sum_{g \geq 0} \lambda^{2 g} \int_{\overline{\mathcal{M}}_{g, l}} \frac{\Lambda_{g}^{\vee}(1)}{\frac{1}{3}\left(\frac{1}{3}-\psi_{1}\right)}=\left(\frac{\sinh (3 \lambda / 2)}{3 \lambda / 2}\right)^{2}, \\
& \sum_{g \geq 0} \lambda^{2 g} \int_{\overline{\mathcal{M}}_{g, l}} \frac{\Lambda_{g}^{\vee}(1)}{\left(1-\psi_{1}\right) \cdot \frac{1}{2}\left(\frac{1}{2}-\psi_{2}\right)}=\frac{4}{3}\left(\frac{\sinh \lambda}{\lambda}\right)^{3}, \\
& \sum_{g \geq 0} \lambda^{2 g} \int_{\overline{\mathcal{M}}_{g, l}} \frac{\Lambda_{g}^{\vee}(1)}{\prod_{j=1}^{3}\left(1-\psi_{j}\right)}=\frac{1}{2}\left(\left(\frac{\sinh (\lambda / 2)}{\lambda / 2)}\right)^{3}\left(\frac{\sinh (3 \lambda / 2)}{3 \lambda / 2}\right)+\left(\frac{\sinh \lambda}{\lambda}\right)^{4}\right) .
\end{aligned}
$$

They can be transferred to results on $C_{n_{1}, \ldots, n_{h}}(p,-p)$ for $n_{1}+\cdots+n_{h} \leq 3$ by (1).

We now indicate how to prove (10) and (11). Write

$$
X=C_{2}(p,-p-1), \quad Y=C_{1,1}(p,-p-1) .
$$

By taking $\alpha=-p$ and $\beta=p$ in (29) one gets

$$
\begin{aligned}
\left(p+\frac{1}{2}\right) C_{2}(-p, p) X-(p+1) C_{1}(-p, p)^{2} Y & \\
& -p C_{1}(p,-p-1)^{2} C_{1,1}(-p, p)+\lambda^{2} p^{2}(p+1) C_{1,1}(-p, p) Y=0 .
\end{aligned}
$$

By changing $p$ to $-p-1$, one gets another linear equation:

$$
\begin{aligned}
\left(p+\frac{1}{2}\right) C_{2}(p+1,-p-1) X-p C_{1}(p+1,-p-1)^{2} Y & \\
-(p+1) C_{1}(-p & -1, p) C_{1,1}(p+1,-p-1) \\
& +\lambda^{2} p(p+1)^{2} \cdot \frac{1}{2} C_{1,1}(p+1,-p-1) Y=0 .
\end{aligned}
$$


We have known from above the coefficients of these equations, hence can solve them to get (10) and (11).

Another important consequence of Theorem 5 is the following:

Theorem 6. [20] For integral $k>0$ we have

(31) $C_{1,1}(k,-1)=\frac{(k-1) ! k !}{(2 k) !}\left(\frac{\sin (\lambda / 2)}{\lambda / 2}\right)^{2 k} \sum_{l=0}^{k-1}\left(\begin{array}{c}2 k-1 \\ l\end{array}\right) \frac{\sin [(2 k-1-2 l) \lambda / 2]}{\sin (\lambda / 2)}$.

It is shown in [20] that (31) is equivalent to the following conjecture of Monni, Song, and Song [15]:

Conjecture 5. For integers $k \geq 1$,

$G(t,-k)=\frac{2(k-1) ! k !}{(2 k) !}\left(\frac{\sin (t / 2)}{t / 2}\right)^{2 k}\left[2^{2(k-2)+1}+\sum_{n=1}^{k-1} \sum_{i=0}^{k-n-1}\left(\begin{array}{c}2 k-1 \\ i\end{array}\right) \cos (n t)\right]$,

where

$$
G(t, k)=\frac{1}{2}+\sum_{g \geq 1} t^{2 g} \sum_{i=0}^{g} k^{i} \int_{\overline{\mathcal{M}}_{g, 2}} \frac{\lambda_{g-i}}{\left(1-\psi_{1}\right)\left(1-\psi_{2}\right)} .
$$

Theorem 6 is proved as follows. Using (29) and (30), we first get

$$
C_{1,1}(\alpha,-1)=\frac{1}{4\left(\alpha-\frac{1}{2}\right)}\left(\frac{\sin (\lambda / 2)}{\lambda / 2}\right)^{2 \alpha}+\frac{\alpha-1}{\alpha-\frac{1}{2}}\left(\frac{\sin \lambda}{\lambda}\right)^{2} C_{1,1}(\alpha-1,-1) .
$$

Since $C_{1,1}(1,-1)$ is known, $C_{1,1}(k,-1)$ can be calculated by induction.

Another application of Theorem 5 is the fact that one can recursively find $C_{2}(\alpha, \beta)$ and $C_{1,1}(\alpha, \beta)$ for all integral $\alpha, \beta$. This is proved in [20] by finding analogues of (32) from (28) - (30). More precisely, suppose $C_{2}(\alpha, \beta)$ and $C_{1,1}(\alpha, \beta)$ has been known for all $\beta$ and some $\alpha$, we derive formulas for $C_{2}(-\alpha, \beta)$, $C_{1,1}(-\alpha, \beta), C_{2}(\alpha-1, \beta)$ and $C_{1,1}(\alpha-1, \beta)$. For example,

$$
\begin{aligned}
& C_{2}(-\alpha,-\beta) \\
& \quad=C_{1}(-\alpha,-\beta)^{2} \frac{\beta K(\alpha, \beta-1) C_{1,1}(\alpha, \beta)-(\beta-1) K(\alpha, \beta) C_{1,1}(\alpha, \beta-1)}{\beta K(\alpha, \beta-1) C_{2}(\alpha, \beta)-\left(\beta-\frac{1}{2}\right) K(\alpha, \beta) C_{2}(\alpha, \beta-1)},
\end{aligned}
$$

and

$$
\begin{aligned}
& C_{1,1}(-\alpha,-\beta) \\
= & C_{1}(-\alpha,-\beta)^{2} \frac{(\beta-1) C_{2}(\alpha, \beta) C_{1,1}(\alpha, \beta-1)-\left(\beta-\frac{1}{2}\right) C_{2}(\alpha, \beta-1) C_{1,1}(\alpha, \beta)}{\beta K(\alpha, \beta-1) C_{2}(\alpha, \beta)-\left(\beta-\frac{1}{2}\right) K(\alpha, \beta) C_{2}(\alpha, \beta-1)},
\end{aligned}
$$

where $K(\alpha, \beta)=C_{1}(\alpha, \beta)^{2}+\lambda^{2} \alpha \beta C_{1,1}(\alpha, \beta)$. Recall $C_{1}(\alpha, \beta)$ has been calculated in previous work [18]. See also (4). Interestingly, Theorem 6 provides the initial values for recursion.

In [20] we also prove similar identities for $C_{3}(\alpha, \beta), C_{2,1}(\alpha, \beta), C_{1,1,1}(\alpha, \beta)$. We will not give their explicit forms here since they are too complicated. This time there are ten types of degree 3 decorated graphs, hence each of the identities has 
ten terms on the left-hand side. To establish (12)-(14), we take suitable choices of $\alpha$ and $\beta$ in two of these identities to get three equations, two of which are linear, one quadratic. It is an elementary exercise to solve them.

It is conceivable that a proof of higher degree Marino-Vafa formula should involve higher degree moduli spaces. In joint work in progress with Liu, generalization to higher degrees are being pursued.

The proofs of Theorem 2, Theorem 5, and Theorem 6 will appear in [20]. Theorem 1 and Theorem 3 are proved by standard combinatorial techniques, their proof will appear in [21]. To end this note, we want to mention that by comparison with Hurwitz numbers, we have derived in [21] a cut-and-join equation for a suitable generating series of the right-hand side of (7), hence also of $C_{n_{1}, \ldots, n_{h}}$ by the Marino-Vafa formula. This equation is used to prove Theorem 4 .

\section{Acknowledgements}

The author thanks Professor Kefeng Liu for bringing his attentions to [9] and [14], and for many discussions.

\section{References}

[1] T. Ekedahl, S. Lando, M. Shapiro, A. Vainshtein, Hurwitz numbers and intersections on moduli spaces of curves. Invent. Math. 146 (2001), 297-327.

[2] C. Faber, Algorithms for computing intersection numbers on moduli spaces of curves, with an application to the class of the locus of Jacobians, New trends in algebraic geometry (Warwick, 1996), 93-109, London Math. Soc. Lecture Note Ser., 264, Cambridge Univ. Press, Cambridge, 1999.

[3] C. Faber, R. Pandharipande, Hodge integrals and Gromov-Witten theory, Invent. Math. 139 (2000), 173-199.

[4] Hodge integrals, partition matrices, and the $\lambda_{g}$ conjecture, preprint: math.AG/9908052

[5] L L L L L L L ithmic series and Hodge integrals in the tautological ring, With an appendix by Don Zagier. Dedicated to William Fulton on the occasion of his 60th birthday. Michigan Math. J. 48 (2000), 215-252.

[6] I. P. Goulden, D. M. Jackson, A. Vainshtein, The number of ramified coverings of the sphere by the torus and surfaces of higher genera, Ann. Comb. 4 (2000), 27-46.

[7] T. Graber, R. Pandharipande, Localization of virtual classes, Invent. Math. 135 (1999), 487-518.

[8] T. Graber, R. Vakil, Hodge integrals and Hurwitz numbers via virtual localization, preprint: math.AG/0003028

[9] S. Katz, C.-C. Liu, Enumerative geometry of stable maps with Lagrangian boundary condtions and multiple covers of the disc, Adv. Theor. Math. Phys. 5 (2001), 1-49.

[10] M. Kontsevich, Intersection theory on the moduli space of curves and the matrix Airy function, Comm. Math. Phys. 147 (1992), 1-23.

[11] Enumeration of rational curves via torus actions, The moduli space of curves (Texel Island, 1994), 335-368, Progr. Math. 129, Birkhäuser Boston, Boston, MA, 1995.

[12] A.-M. Li, G. Zhao, Q. Zheng, The number of ramified coverings of a Riemann surface by Riemann surface, Comm. Math. Phys. 213 (2000), 685-696.

[13] I. G. Macdonald, Constant term identities, orthogonal polynomials, and affine Hecke algebras, Doc. Math. 1998, Extra Vol. I, 303-317.

[14] M. Marino, C. Vafa, Framed knots at large N, preprint: hep-th/0108064 
[15] S. Monni, J. S. Song, Y.S. Song, The Hurwitz enumeration problem of branched covers and Hodge integrals, preprint: hep-th/0009129

[16] D. Mumford, Towards an eunumerative geometry of the moduli space of curves, Arithmetic and geometry, Vol. II, 271-328, Progr. Math. 36, Birkhäuser Boston, Boston, MA, 1983.

[17] A. Okounkov, R. Panharipande, Gromov-Witten theory, Hurwitz numbers, and Matrix models. I, preprint: math.AG/0101147

[18] G. Tian, J. Zhou, Quadratic recursion relations of Hodge integrals via localization, to appear in Mathematicae Sinica.

[19] E. Witten, Two-dimensional gravity and intersection theory on moduli space, Surveys in differential geometry (Cambridge, MA, 1990), 243-310, Lehigh Univ., Bethlehem, PA, 1991.

[20] J. Zhou, On some conjectures for Hodge integrals, in preparation.

[21] _ Hodge integrals, Hurwitz numbers, and symmetric groups, in preparation.

Department of Mathematical Sciences, Tsinghua University, Beijing, 100084, China.

E-mail address: jzhou@math.tsinghua.edu.cn 